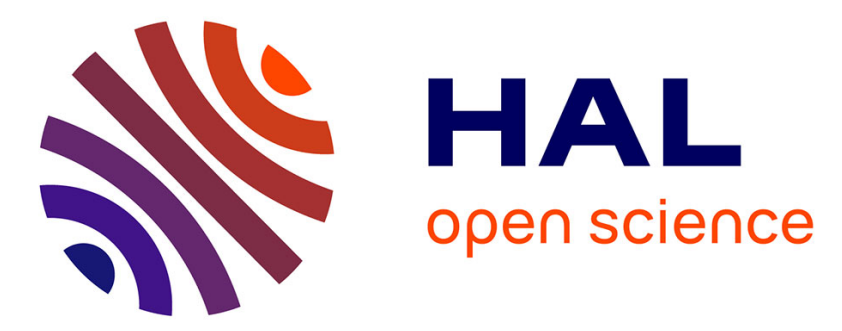

\title{
Les estampilles littérales et anépigraphes sur céramique belge et le rapport à l'écrit des potiers belgo-romains Xavier Deru
}

\section{To cite this version:}

Xavier Deru. Les estampilles littérales et anépigraphes sur céramique belge et le rapport à l'écrit des potiers belgo-romains. Gallia - Archéologie de la France antique, 2004, L'écriture dans la société gallo-romaine, 61, pp.133-143. 10.3406/galia.2004.3190 . hal-01910975

\section{HAL Id: hal-01910975 \\ https://hal.science/hal-01910975}

Submitted on 29 Jan 2020

HAL is a multi-disciplinary open access archive for the deposit and dissemination of scientific research documents, whether they are published or not. The documents may come from teaching and research institutions in France or abroad, or from public or private research centers.
L'archive ouverte pluridisciplinaire HAL, est destinée au dépôt et à la diffusion de documents scientifiques de niveau recherche, publiés ou non, émanant des établissements d'enseignement et de recherche français ou étrangers, des laboratoires publics ou privés.

\section{(ㅇ)(1) $\$$}

Distributed under a Creative Commons Attribution - NonCommercial - NoDerivatives $\mid 4.0$ 


\title{
LES ESTAMPILLES LITTÉRALES ET ANÉPIGRAPHES SUR CÉRAMIQUE BELGE ET LE RAPPORT À L'ÉCRIT DES POTIERS BELGO-ROMAINS
}

\author{
Xavier DERU
}

Mots-clés. Gaule belgique, $r^{r}$ s., artisanat, potiers, terra rubra, terra nigra, estampille.

Résumé. La céramique belge correspond à deux catégories (terra rubra et terra nigra) et comprend un répertoire de vaisselle de table issu de la terre sigillée italique, de la céramique celtique et de la vaisselle métallique. Après une caractérisation globale de l'estampillage de cette céramique, l'estampillage spécifique à certaines régions (Champagne, Moselle et Rhénanie, Nord) est examiné sous l'angle de la chronologie, du répertoire et de la qualité. Les formes de l'estampillage (épigraphique ou non, avec cadre, ligature, abréviation, etc.) dépendent des potiers, de leur origine et de leur dépendance aux répertoires imités, ainsi que de leurs exigences et de leur environnement. En Champagne et dans les villes de Moselle, les potiers qui imitent la terre sigillée précoce montrent une proximité avec la culture méditerranéenne de l'écrit, de même que les potiers septentrionaux qui s'inspirent de produits métalliques dans la seconde moitié du r" s. Pour d'autres productions régionales, si l'estampillage reste une norme, il perd toute signification; toutefois, il témoigne d'une référence à l'écrit que la grande majorité des produits n'exprime pas.

Key-words. Gallia Belgica, craft, potters, terra rubra, terra nigra, stamp.

Abstract. Belgic pottery which includes terra rubra and terra nigra has a repertoire of tableware forms stemming from Italic samian ipottery, Celtic pottery and metal ware. An overall characterization of stamps is defined and specific stamps for several regions are being analyzed (Champagne, Moselle and Rhineland, Northern Gaul) from three angles: chronology, patterns and qualit). The forms of stamps (spigraphic or not, with border, ligature, abbreviation, etc.) depended on the potters, on their origin and the patterns imitated, as on their requirements and on the environment. In Champagne and in the towns of Moselle potters copy early samian pottery and show close links with the Mediterranean writing culture, as Northern potters who take models from metal products in the second half of the $1^{\text {st }}$ century. In other regional productions, stamping is still the norm and if it looses all sense it attests however a reference to zuriting that the vast majority of products doesn't express.

Translation : Isabelle FALDLTT

Schlagwörter. Gallia Belgica, 1. Jahrh. n. Chr., Handwerk, Töpfer, terra rubra, terra nigra, Stempel.

Zusammenfassung. Die beiden Hauptgruppen der sogenannten Belgischen Ware (terra rubra und terra nigra) umfassen Formen des Tafelgeschirrs, die aus dem Formenrepertoire der italischen Sigillata, der keltischen Keramik und dem des Metallgeschirrs entlehnt sind. Der Beitrag bietet eine Darstellung der Stempeltypen dieser Keramik und untersucht regionsspezifische Stempelarten (Champagne, Moselund Rheinlande, Norden) hinsichtlich ihrer Chronologie, der Zusammensetzung ihres Repertoires und ihrer Qualität. Die Art der Stempel (epigraphisch oder anepigraphisch, mit Rahmen, Ligaturen, Abkürzungen, etc.) hängt ebenso von der Herkunft der Töpfer und deren Abhängigkeit vom nachgeahmten Repertoire ab wie von ihren eigenen Bedürfnissen und ihrem L'mfeld. Bei in der Champagne und den Moselstädten Töpfer, die frühe Sigillata imitieren, zeigt sich eine große Nähe zur mediterranen Schriftkultur. Die nördlichen Töpfer holen sich in der zueiten Hälfte des 1. Jahrh. $n$. Chr. Anregungen bei der Metallproduktion. Wo bei anderen Regionalproduktionen Stempelung zur Norm wird, verliert sie mit der Zeit jegliche besondere Bedeutung. Dennoch bleibt sich hier ein Bezug ablesbar, den die große Mehrzahl der übrigen Produkte nicht erkennen läßt.

Übersetzung : Stefan WIRTH

L'épigraphie de l'instrumentum sert la connaissance de l'artisanat antique, spécifiquement les problématiques touchant à la production (voir Bémont, supra, p. 103-131) et à la commercialisation (voir Delage, infra, p. 145-152). C'est d'ailleurs par le biais de ces inscriptions que l'artisanat fut d'abord appréhendé. D'un point de vue macro-économique et sociologique, ce corpus pourrait également témoigner de "l'imprégnation de l'écrit " de la société romaine ; l'écrit étant pratiqué dans toutes les activités et par toutes les classes de cette société. Toutefois, disparités sociales et culturelles, géographiques et chronologiques, sont constitutives de l'histoire; nous ne pouvons donc traiter ce phénomène de manière simple et globale, " suivant le 
diktat des sources ". Prudemment, je voudrais mettre en lumière à la fois les facteurs qui rendent cette référence à l'écrit explicite et les éléments qui en indiquent la proximité, cela à partir d'une source bien circonscrite, la céramique belge et ses estampilles littérales et anépigraphes.

Évitant de nous écarter de cette discussion, nous n'exploiterons pas cette source dans une perspective onomastique ou économique. Le corpus documentaire comprend actuellement 4200 estampilles répertoriées dans une base de données informatisée. Loin d'être achevé, ce travail demande encore de nombreux compléments et corrections. Toutefois, dans le cadre fixé, le répertoire offre une documentation représentative, même si nous nous risquerons rarement à des quantifications précises.

Après avoir présenté rapidement le support estampillé, nous traiterons nos sources au moyen d'une division en trois de la Gaule septentrionale : la région champenoise, la Moselle et le Rhin, et le Nord. Cette division a priori s'appuie sur l'intuition que ces régions témoignent de faciès artisanaux et sociaux différents ; ces notions seront nuancées par la suite. À l'intérieur de ces espaces, le mobilier issu d'ateliers sera privilégié car il témoigne au mieux de l'artisanat régional ${ }^{243}$. Dans un deuxième temps, nous exploiterons les données de manière diachronique pour déboucher ensuite sur une synthèse.

\section{LA CÉRAMIQUE BELGE}

Les catégories de céramique faisant l'objet d'un estampillage ne sont guère nombreuses eu égard à la variété de la céramique romaine. La terre sigillée et les amphores sont certes les catégories offrant les plus riches corpus; les mortiers, la céramique à vernis rouge pompéien, les dolia présentent des catalogues plus réduits. En Gaule romaine, les catégories précoces de vaisselle de table (céramique fumigée, imitation de terre sigillée helvétique et céramique belge), qui dépendent de la terre sigillée de type italique pour une part de leur répertoire morphologique, font également l'objet d'un estampillage. Ces trois dernières catégories de céramique gallo-romaine partagent le territoire situé au nord des Alpes en trois régions inégales : la Gaule centrale et occidentale, le plateau Suisse et la Gaule septentrionale. Elles pourraient paraître artificielles et simplement dues à l'historiographie, mais en fait, ces catégories correspondent bel et bien à des faciès typologiques distincts. Elles témoignent cependant d'un même phénomène, comprenant d'une part celui de la romanisation de l'artisanat céramique et d'autre part celui de l'art de la table. En outre, la céramique belge qui se retrouve entre la Seine et le Rhin est celle qui rassemble, de loin, le plus grand nombre d'estampilles.

D'un point de vue technique, la céramique belge se partage en deux catégories, selon la couleur de la surface obtenue par la cuisson soit oxydante (mode A), soit réductrice (mode B) : la terra rubra (TR) et la terra nigra (TN) (Deru, 1996a, p. 19-23).

243. Pour les estampilles provenant de sites de consommation, mais attribuées à un atelier ou à une région de production, nous avons régulièrement validé cette attribution par une observation de la pâte des céramiques.
Des catégories plus fines ont été caractérisées d'après le traitement de leur surface : le lissage, l'engobage et l'enfumage.

Terra rubra et terra nigra présentent un répertoire morphologique commun, mais également des typologies spécifiques à l'une et à l'autre (fig. 73) (Deru, 1996a, p. 28-149). Les formes se répartissent en assiettes et plats, coupes, bols, calices, pots et bouteilles. Aucune estampille ne fut reconnue sur des calices et des bols, et les bouteilles en portent de manière exceptionnelle (moins de 0,5\%). Ce sont donc les assiettes (65\%), les coupes (22\%) et les pots ( $12 \%$ ) qui fournissent le plus grand nombre d'estampilles. Presque toutes les formes d'assiettes et de coupes comportent des exemplaires estampillés, mais l'estampillage n'est jamais systématique. Nous pouvons partager le répertoire des assiettes et des coupes en deux groupes, le premier avec les formes les plus précoces (10 av. J.-C./50 apr. J.-C.) qui imitent principalement la terre sigillée de type italique (A1, A5-9, A17-18, A24-25, C8 et C13) et le second groupe qui rassemble des assiettes sans prototype direct, généralement plus récentes (50-100 apr. J.-C.) et uniquement produites en terra nigra (A38-39 et A41-43). La diversité des pots qui font l'objet d'un estampillage est bien moindre et cet usage varie selon les régions. Ainsi les groupes typologiques précoces, les pots à lèvre oblique (Pl-12), les pots-tonnelets (P13-19) et ceux à lèvre en amande (P20-22) en sont dépourvus à une exception près (voir infra). Les pots issus du répertoire celtique (P36-40), comme les bouteilles, ne sont pas marqués non plus. En fait, seuls les pots à col concave et lèvre effilée (P41-53) et les pots biconiques (P54-56) sont régulièrement estampillés.

\section{LES ESTAMPILLES}

Les estampilles ont déjà fait l'objet d'une caractérisation sur la base de critères externes qu'il n'y a pas lieu de remettre à jour (Deru, 1996a, p. 155-158). Nous reviendrons plus en détail sur quelques points dans la suite de cet article. Rappelons donc que les estampilles sont généralement rectangulaires et comportent le plus souvent une simple ligne et quelquefois deux. Les autres formes, circulaires, courbes ou in planta pedis, sont beaucoup plus rares (au total $5 \%$ ).

Les estampilles rectangulaires doubles, les circulaires et celles in planta pedis se trouvent au centre des formes ouvertes. Les estampilles rectangulaires peuvent également être imprimées sur le fond externe des formes fermées. Les estampilles courbes, quant à elles, se situent toujours à cet endroit.

Les estampilles littérales se définissent comme des associations intelligibles de caractères alphabétiques. D'un point de vue graphique, les estampilles non littérales sont plus complexes à saisir et seront développées plus loin, mais de celles-ci nous excluons les estampilles iconographiques présentant des motifs simples : protomés de cheval, aigle, lièvre, etc.

\section{LA CHAMPAGNE}

La Champagne, c'est-à-dire l'étroite région calée sur les vallées de la Vesle et de la Marne, à hauteur de la montagne de Reims, regroupe au $\mathrm{I}^{\mathrm{er}} \mathrm{s}$. un grand nombre de sites de produc- 

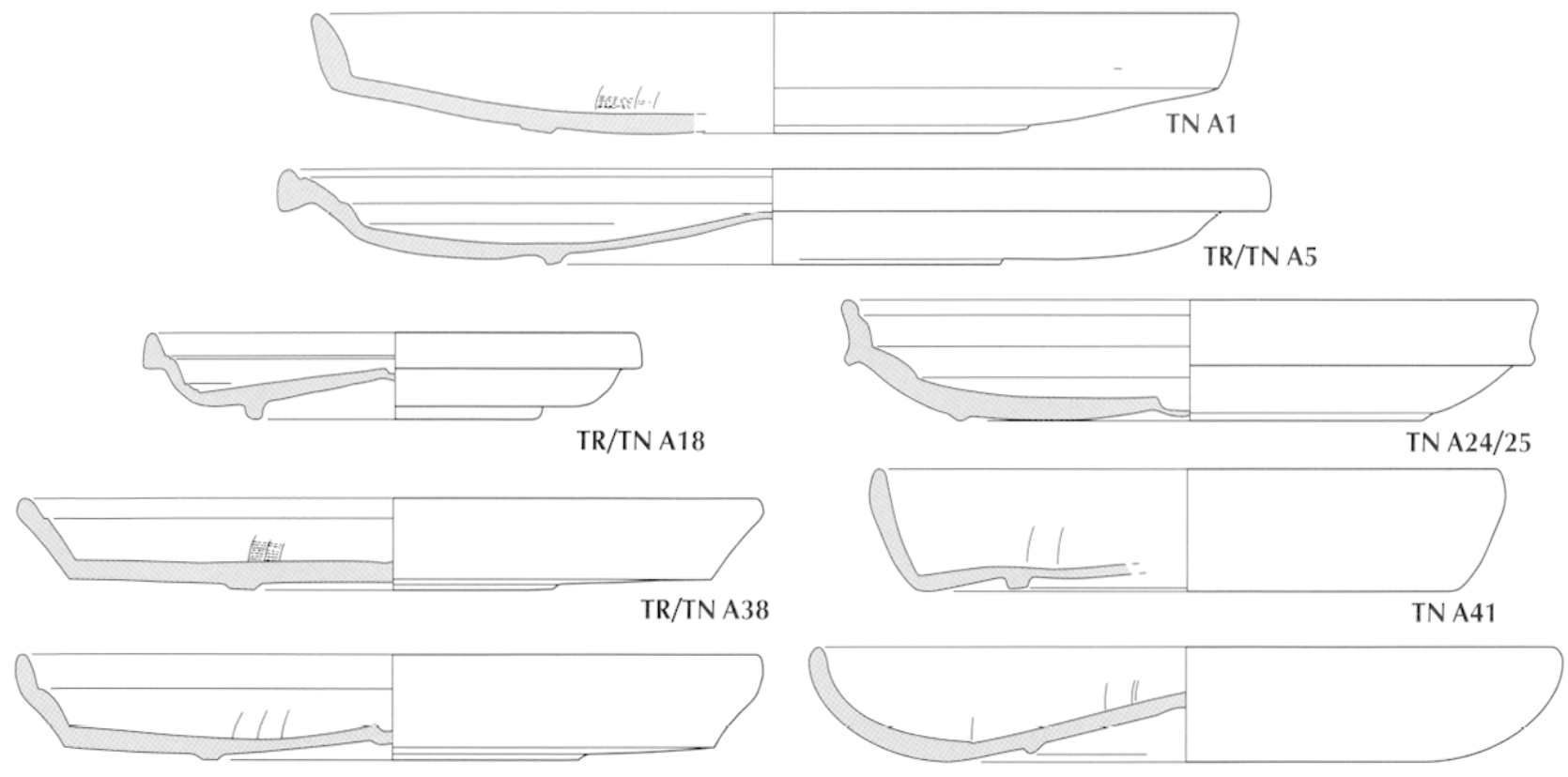

TN A39

TN A42
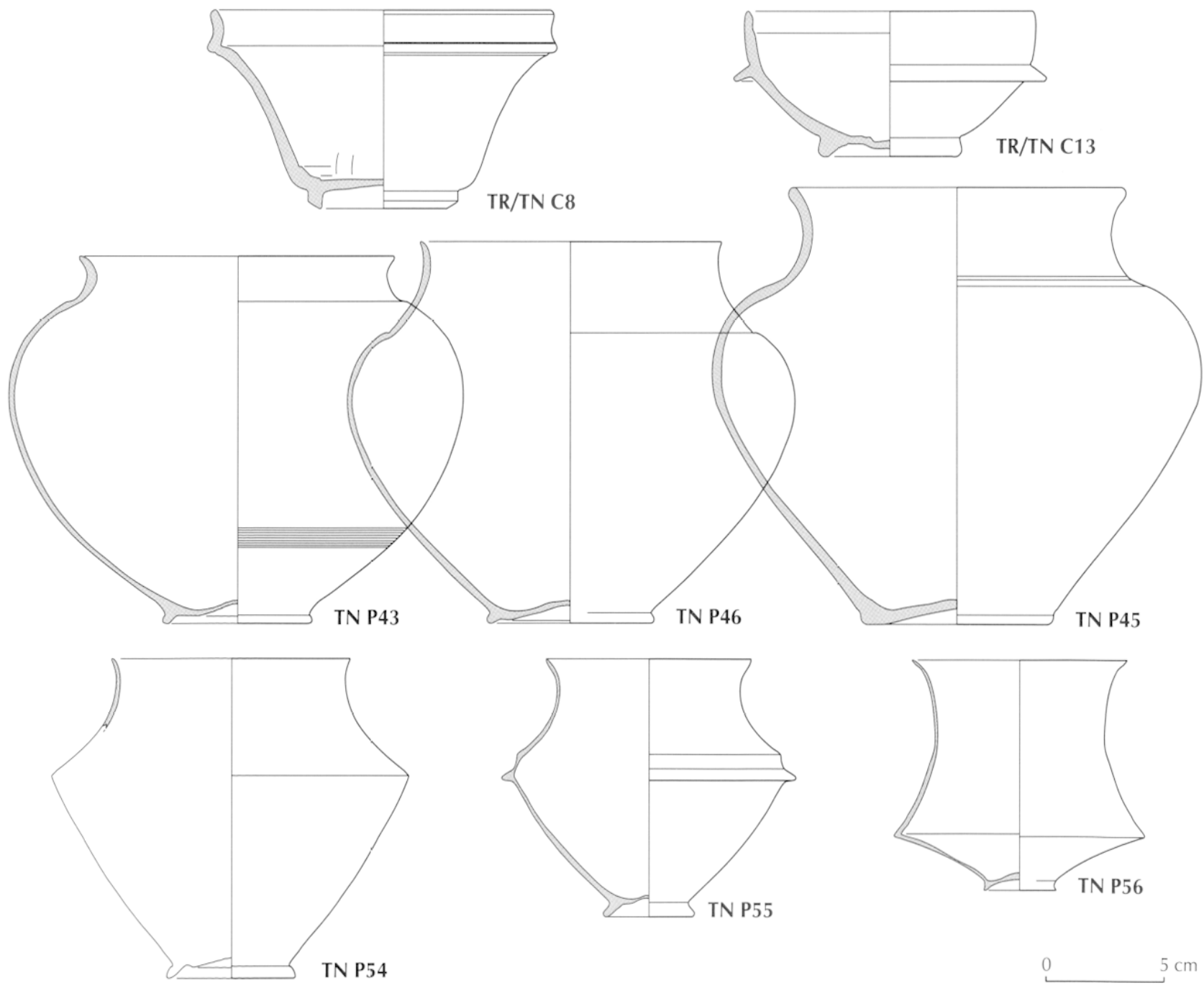

Fig. 73 - Principales formes de terra rubra (TR) et de terra nigra (TN) estampillées (DAO:X. Deru, CNRS). 


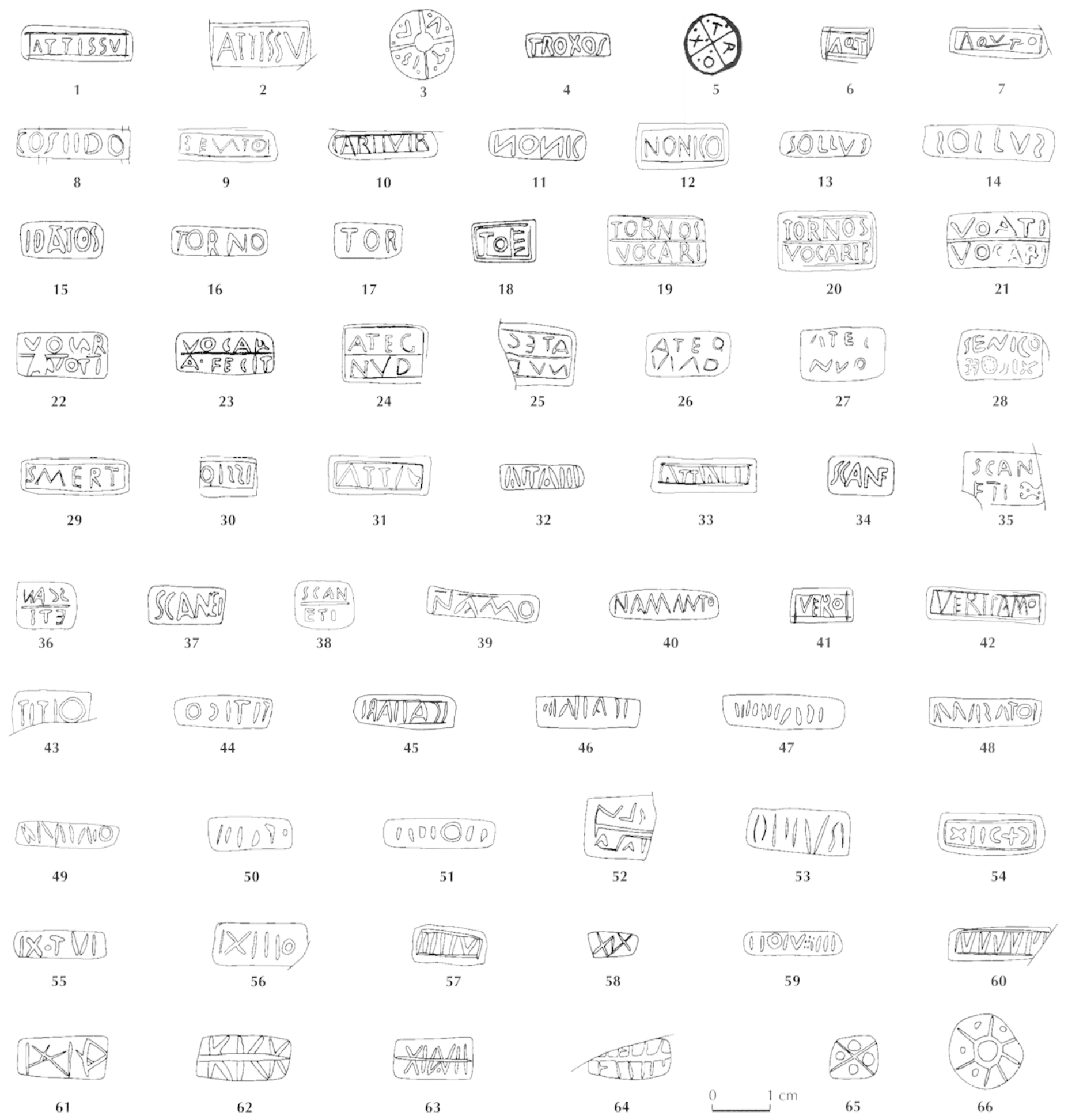

Fig. 74 - Estampilles sur terra rubra (TR) et terra nigra (TN) fabriquées en (Champagne (cf. lableau, p. 137).

tion de céramique belge, ce qui signifie d'un point de vue économique une multitude d'ateliers et d'unités de production ${ }^{244}$. Au-delà du $\mathrm{I}^{\mathrm{cr}} \mathrm{s}$., plusicurs de ces ateliers poursuivent leurs activités par la fabrication de céramique rugueuse sombre.

244. Pour le catalogue de l'ensemble des ateliers, voir Deru, 1996a, p. 263-317. Pour la Champagne (Deru, 1999), un atlas des ateliers est actuellement réalisé avec la collaboration de M. Chossenot.

\section{LES ATELIERS ET LES PRODUITS}

Reims, la capitale régionale, abritait un quartier réservé au travail de la terre, le quartier Saint-Remi. Malgré les carences de la documentation, on considere ce centre de production de céramique belge comme le plus important d'un point de vuc économique. Trois autres ateliers ont récemment complété nos connaissances : un atelier découvert boulevard de la Paix vers 


\begin{tabular}{|c|c|c|c|}
\hline \multicolumn{4}{|c|}{ FIGURE 74 (suite) } \\
\hline $\mathrm{N}^{\circ}$ & Site (Pays) & Forme & Bibliographie \\
\hline 1 & $\operatorname{Reims}(F)$ & TR1a A & Deru, Grasset, 1997 \\
\hline 2 & Saint-Martin-Longueau (F) & TR1b A & Arch. pers. \\
\hline 3 & Onnaing (B) & TR1a C8 & Arch. pers. \\
\hline 4 & Trèves (D) & TN A7 & Koethe, 1938 \\
\hline 5 & Blicquy (B) & TN A5 & De Laet, Van Doorselaer et al., 1972 \\
\hline 6 & Reims (F) & TR2 C8 & Deru, Grasset, 1997 \\
\hline 7 & $\operatorname{Reims}(F)$ & TR1a A & Deru, Grasset, 1997 \\
\hline 8 & Andernach (D) & TN A5 & Arch. pers. \\
\hline 9 & Bavay (F) & TN A18 & Arch. pers. \\
\hline 10 & Saint-Martin-Longueau (F) & TR A & Arch. pers. \\
\hline 11 & Bavay (F) & TR1a A18 & Loridant, Deru, à paraitre \\
\hline 12 & Andernach (D) & TR2 A17 & Arch. pers. \\
\hline 13 & Courmelois (F) & TNA & Arch. pers. \\
\hline 14 & Courmelois (F) & TN A41 & Arch. pers. \\
\hline 15 & Nimègue (NL) & TR A5 & Holwerda, 1941 \\
\hline 16 & Trépail (F) & TN C8 & Deru, Redon, Louvion, 2003 \\
\hline 17 & Trépail (F) & TN C8 & Deru, Redon, Louvion, 2003 \\
\hline 18 & Gatesbury (GB) & TN C8 & Partridge, Rigby, 1981 \\
\hline 19 & Wederath (D) & TN A41 & Haffner, 1978 \\
\hline 20 & Saint Albans (GB) & TN A31 & Stead, Rigby, 1989 \\
\hline 21 & Trèves (D) & TR A18 & Koethe, 1938 \\
\hline 22 & Urmitz (D) & TR2 C13 & Arch. pers. \\
\hline 23 & Nimègue (NL) & TN A18 & Holwerda, 1941 \\
\hline 24 & Andernach (D) & TR A14 & Arch. pers. \\
\hline 25 & Reims (Г) & TR A & Brusel, Deru, en préparation \\
\hline 26 & Reims (F) & TN A17 & Deru, Rollet, 2000 \\
\hline 27 & Xanten (D) & $T R / T N A$ & Arch. pers. \\
\hline 28 & Trépail (F) & TR/TN & Deru, Redon, Louvion, 2003 \\
\hline $29 / 30$ & Reims (F) & TR1a & Deru, Grasset, 1997 \\
\hline 31 & Andernach (D) & TR A 18 & Arch. pers. \\
\hline 32 & Urmitz (D) & TR C & Arch. pers. \\
\hline 33 & Andernach (D) & TR2 A5 & Arch. pers. \\
\hline 34 & Nimègue (NL) & TR C8 (?) & Holwerda, 1941 \\
\hline 35 & Nimègue (NL) & TR1b A14 & Arch. pers. \\
\hline 36 & Nimègue (NL) & TR1b C8 & Arch. pers. \\
\hline 37 & Saint Albans (GB) & TR1b C8 & Stead, Rigby, 1989 \\
\hline 38 & Saint Albans (GB) & TR1a C8 & Stead, Rigby, 1989 \\
\hline 39 & Reims (F) & TN A41-42 & Arch. pers. \\
\hline 40 & $\operatorname{Reims}(F)$ & TN A5 & Brusel, Deru, en préparation \\
\hline 41 & $\operatorname{Reims}(\mathrm{F})$ & TR2 C8 & Deru, Grasset, 1997 \\
\hline 42 & $\operatorname{Reims}(F)$ & TR1a A & Arch. pers. \\
\hline 43 & Courmelois (F) & TNA & Deru,1996a, p. 280-281 \\
\hline 44 & Courmelois (F) & TN A39 & Deru, 1996a, p. 280-281 \\
\hline 45 & Arlon (B) & TR1a ^18 & Arch. pers. \\
\hline 46 & Bavay (F) & TR1a A31 & Loridant, Deru, à paraître \\
\hline 47 & Bavay (f) & TR1a A31 & Loridant, Deru, à paraître \\
\hline 48 & Xanten (D) & TNA & Arch. pers. \\
\hline 49 & Bavay (F) & TNA & Loridant, Deru, à paraitre \\
\hline 50 & Tours-sur-Marne (F) & TN A & Deru, $1996 \mathrm{c}$ \\
\hline 51 & Courmelois (F) & TN A41 & Deru, 1996a, p. 280-281 \\
\hline 52 & $\operatorname{Reims}(F)$ & TNA & Deru, Grasset, 1997 \\
\hline 53 & $\operatorname{Reims}(F)$ & TR3 P7 & Deru, Rollet, en préparation \\
\hline 54 & Tours-sur-Marne (F) & TNA & Deru, 1996c \\
\hline 55 & Courmelois (F) & TN A39 (?) & Deru, 1996a, p. 280-281 \\
\hline 56 & Trépail (F) & TNA & Deru, Redon, Louvion, 2003 \\
\hline 57 & Thérouanne (F) & $\mathrm{TNC} 13$ & Deru, Thuillier, 2001 \\
\hline 58 & Bavay (F) & TR C8 & Loridant, Deru, à paraître \\
\hline 59 & Reims (F) & TNC & Deru, Grasset, 1997 \\
\hline 60 & Sept-Saulx (i) & TR A18 & Deru, 1996a, p. 308-309 \\
\hline 61 & Sept-Saulx (F) & TNA & Deru, 1996a, p. 308-309 \\
\hline 62 & Bavay (F) & TR1aC13 & Loridant, Deru, à paraître \\
\hline 63 & Sept-Saulx (F) & TN C8 & Deru, 1996a, p. 308-309 \\
\hline 64 & Tours-sur-Marne (F) & TNA & Deru, $1996 c$ \\
\hline 65 & Courmelois (F) & TNC3 & Deru, 1996a, p. 280-281 \\
\hline 66 & Courmelois (F) & TN A & Deru, 1996a, p. 280-281 \\
\hline
\end{tabular}

1960, mais dont le mobilier avait été oublié dans les réserves du musée Saint-Remi, et deux autres fouillés récemment rue de l'Équerre, l'un en 2000, l'autre en 2001.

Dans le monde rural, les ateliers semblent former de petites unités isolées: Avenay-Val-d'Or, Champillon, Juvigny, Livry "Crottenct ", Louvercy, Mourmelon-le-Petit, Sept-Saulx et Thuisy. Seuls les ateliers de Livry "Champ Dameron " et de Tours-sur-Marne pourraient appartenir à de petites agglomérations. Un nouveau site fut fortuitement découvert à Trépail, dans la montagne de Reims. Cent quatre-vingt-sept chablis, arrachés à la suite de la tempête de l'hiver 1999, ont en effet révélé un abondant matériel qu'un relevé systématique permit d'identifier comme étant un vaste centre de production courrant 22 ha (Deru et al., 2003).

À partir d'observations minéralogiques, les estampilles sur céramique belge que l'on peut attribuer à la Champagne sont de loin plus abondantes que celles trouvées en contexte de production. Il est donc certain que plus nombreux sont les potiers ayant travaillé à Reims ou à Trépail et plus nombreux sont les ateliers ayant produit du matériel estampillé.

Sur les quatorze sites de production, dix ont livré de la vaisselle estampillée. Les formes produites et estampillées sont dominées par les imitations de terre sigillée, aussi bien des assiettes (TR/TN A3-37) que des coupes (TR/TN C2-19). Ces productions caractérisent les horizons de la première moitié du $\mathrm{I}^{\text {er }} \mathrm{s}$.; les assiettes précoces et celles qui appartiennent aux horizons plus récents (TN A39 et A42) complètent le répertoire estampillé. En Champagne, l'estampillage suit l'usage de la terre sigillée et ni les pots ni les bouteilles ne sont marqués. Il convient cependant de noter une exception dans l'atelicr 2001 de la rue de l'Équerre à Reims, il s'agit d'estampilles sur l'épaule de pots en terra rubra enfumée : TR3 P6 (fig. 74, n53).

\section{LES ESTAMPILLES}

Si l'estampillage des assiettes et des coupes est très fréquent en Champagne, il n'est pas systématique. À l'heure actuelle, on ne dispose pas de données pour évaluer la proportion de récipients estampillés pour un même type.

En revanche, il est clair que la majorité des estampilles champenoises sont littérales (fig. 74, $\mathrm{n}^{\circ} 1-49$ ), $15 \%$ environ sont anépigraphes. D'ailleurs, tous les ateliers de Champagne ayant fourni du matériel marqué comportent une majorité d'estampilles littérales. Concernant la forme des estampilles, la Champagne n'a pas livré d'estampille courbe ou in planta pedis; $98 \%$ sont rectangu- laires, dont $10 \%$ sont doubles $\left(\mathrm{n}^{\circ \mathrm{s}} 19-28,35-38,52,62-64\right)$, les $2 \%$ restant sont circulaires ( $\left.n^{\circ} 3,5,66\right)$. On peut considérer qu'un tiers des estampilles rectangulaires sont gravées à l'intérieur d'un cartouche $\left(\mathrm{n}^{\circ \times} 1\right.$, $2,6,7,9,10$, etc.).

D'un point de vue épigraphique, les estampilles littérales de Champagne sont les plus riches et les plus soignées. Les caractères sont, en grande majorité, latins et lisibles. Néanmoins, rares sont ceux qui présentent des empattements aux extrémités des caractères. Ces artifices concernent principalement les lettres $\mathrm{A}, \mathrm{M}, \mathrm{N}$ et $\mathrm{T}$, mais à cause de leur petite taille, ces traits sont difficiles à rendre graphiquement $\left(n^{\omega *} 2,20,24,29\right)$. Plus fréquentes, au contraire, sont les simplifications de caractères: des traits droits, verticaux ou obliques, pour les lettres $A$, E, F et $S\left(n^{\circ s} 1,8,10,31-33,45-47\right)$. Quelques lettres sont écrites de manière rétrograde, en particulier les lettres $\mathrm{N}$ et $\mathrm{S}\left(\mathrm{n}^{\circ \%} 9\right.$, $11,14)$, mais seules quelques marques sont entièrement rétrogrades ( $\mathrm{n}^{\mathrm{os}} 25,36,44,45,53$ ?). On doit néanmoins noter l'cxistence de deux estampilles en boustrophédon ( $n^{\circ} 28$ et $29 / 30$ ). Enfin, les ligatures, bien qu'elles restent rares, sont plus fréquentes en Champagne qu'ailleurs $\left(\mathrm{n}^{\mathrm{os}} 15,18,31,34,37\right)$.

Les estampilles littérales consistent généralement en un nom de potier, rarement deux, associé quelquefois (moins de $10 \%$ ) au verbe latin ou celte, fecit ou avotis, ou à leurs abrévia- 


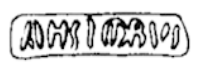

1
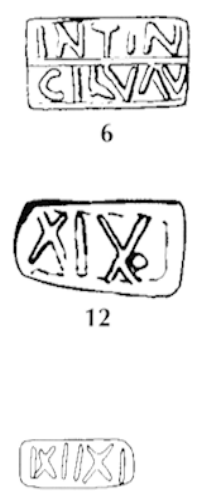

18

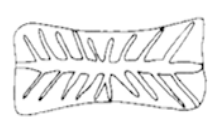

24

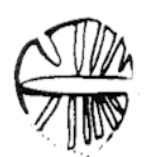

29

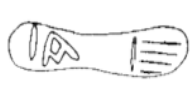

30

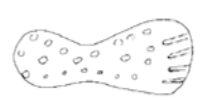

34

2
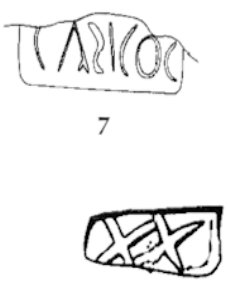

13

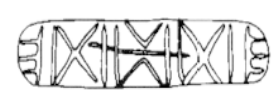

19
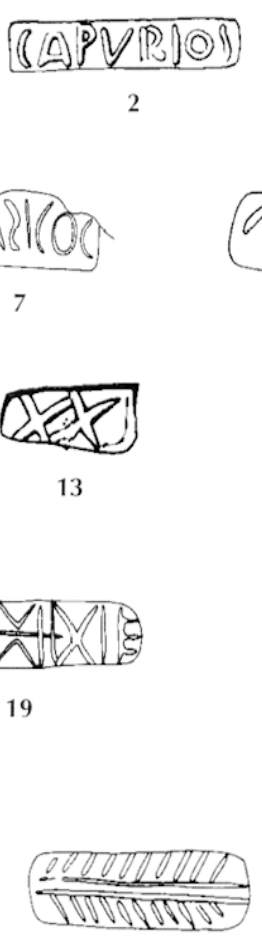

25
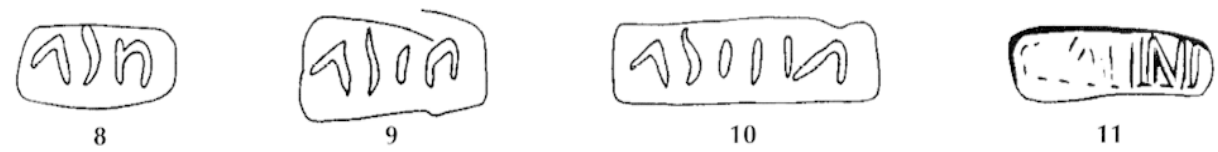

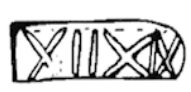

14

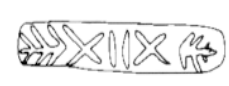

20

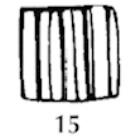

15
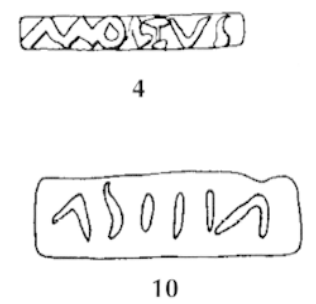

4

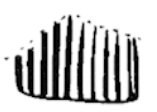

16

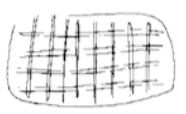

22
Q02

5

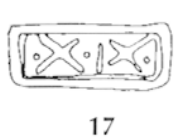

17

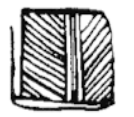

23

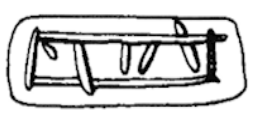

27
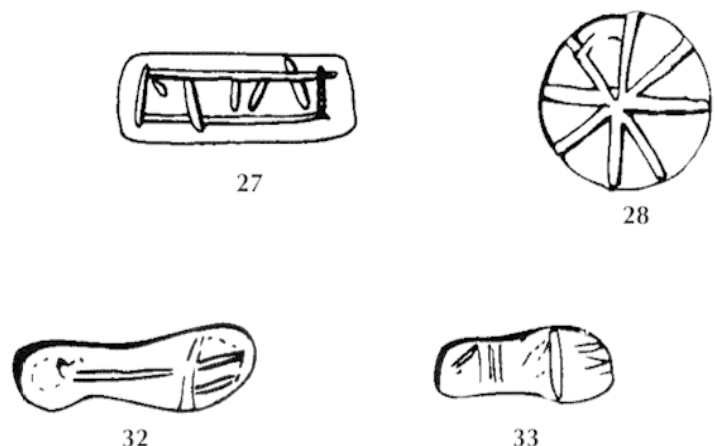

33

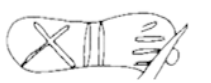

36

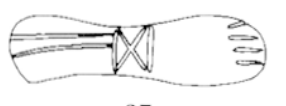

37

Fig. 75 - Estampilles sur terra rubra (TR) et terra nigra (TN) fabriquées en Moselle et en Rhénanie (cf. tableau, p. 139).

tions ( $\mathrm{n}^{\mathrm{os}} 18,20,23,28,31-34$ et 21,22 ) (Lambert, 2002, p. 33-78). Même si, comme nous l'avons dit, nous laissons de côté l'étude onomastique, ces noms témoignent de la maîtrise de l'écrit par les abréviations. Le fait de retrouver au sein d'un même atelier des estampilles présentant des lettres communes constitue un argument solide pour de telles identifications. Par exemple dans l'atelier Saint-Remi de Reims, on a retrouvé plusieurs graphies pour Attissus, Aquto et à Trépail, pour Tornos ( $\left.{ }^{o s} 1-3,6-7,16-20\right)$. Dans le cas où le contexte de découverte n'est pas décisif, c'est la similitude des lettres et du style qui conduit à cette hypothèse, comme pour Canicos, Nonicos ou Troxos ( $\left.{ }^{\circ s} 4-5,11-12\right)$. À partir des mêmes arguments, on pourrait également isoler une autre forme de dénomination, celle des diminutifs. Nous proposons une telle hypothèse pour Namo, Titio et Vero, respectivement pour Namanto de Trépail, Titico de Courmelois et Veritamo de Reims (?) ( $\mathrm{n}^{\text {os }} 39-40$, 41-42, 43-44).

L'observation des estampilles non-littérales complète la caractérisation de l'estampillage en Champagne. Toutefois, il faut préciser qu'une estampille illisible peut être issue d'une estampille littérale, soit mal imprimée, soit due à un cachet dégradé ( $\left.{ }^{\circ s} 45-47,48-49\right)$; nous avons essayé d'éviter cet écueil. Mais ce manque de soin a son intérêt en tant que tel. Ainsi, les estampilles ${ }^{\text {os }} 50-53$, si elles présentent des silhouettes de lettres, sont toutefois dépourvues de signification. Les estampilles suivantes présentent des caractères clairs, mais non intelligibles. On y reconnaît des $\mathrm{O}, \mathrm{I}, \mathrm{V}, \mathrm{T}$ et $\mathrm{X}$, certaines d'entre elles sont encadrées ( $\left.n^{\circ 5} 54-59\right)$. Les $n^{\text {s }} 60-64$ sont plus complexes, les caractères sont peu évidents, surtout lorsque le potier a tenté une estampille double. Les deux dernières estampilles sont clairement décoratives ( $\left.n^{\circ} 65-66\right)$.

Ces deux estampilles proviennent du même atelier, Courmelois, qui compte également les n"s 51 et 55. Sept-Saulx ( $\left.n^{\circ} 60,61,63\right)$, Tours-sur-Marne $\left(n^{\circ 5} 50,65\right)$, Trépail $\left(n^{\circ} 56\right)$ et Louvercy sont d'autres ateliers ruraux où des estampilles nonlittérales ont été retrouvées. Cette ruralité ne semble pas être un critère prépondérant pour expliquer leur fréquence, car les ateliers de Saint-Remi et de la rue de l'Équerre à Reims en 


\begin{tabular}{|c|c|c|c|}
\hline \multicolumn{4}{|c|}{ FIGURE 75 (suite) } \\
\hline $\mathbf{N}^{\circ}$ & Site (Pays) & Forme & Bibliographie \\
\hline 1 & Trèves (D) & TN A5 & Koethe, 1938 \\
\hline 2 & Trèves (D) & TN A41 & Koethe, 1938 \\
\hline 3 & Trèves (D) & TR A18 & Koethe, 1938 \\
\hline 4 & Trèves (D) & TNA & Koethe, 1938 \\
\hline 5 & Trèves (D) & TNA7 & Koethe, 1938 \\
\hline 6 & Trèves (D) & TNA & Koethe, 1938 \\
\hline 7 & Metz (F) & TRA & Reusch, 1943 et arch. pers. \\
\hline 8 & Nimègue (NL) & TN A41-42 & Holwerda, 1941 \\
\hline 9 & Nimègue (NL) & TN A41-42 & Holwerda, 1941 \\
\hline 10 & Nimègue (NL) & TN A41-42 & Holwerda, 1941 \\
\hline 11 & Karden (D) & TNA & Nickel, 1999 \\
\hline 12 & Karden (D) & TNA & Nickel, 1999 \\
\hline 13 & Karden (D) & TNA & Nickel, 1999 \\
\hline 14 & Karden (D) & TNA & Nickel, 1999 \\
\hline 15 & Karden (D) & TNA & Nickel, 1999 \\
\hline 16 & Karden (D) & TNA & Nickel, 1999 \\
\hline 17 & Lebach (D) & TN A41-42 & Gerlach, 1976-1986 \\
\hline 18 & Lebach (D) & TN A41-42 & Gerlach, 1976-1986 \\
\hline 19 & Urmitz (D) & TN A24 & Arch. pers. \\
\hline 20 & Urmitz (D) & TN A25 & Arch. pers. \\
\hline 21 & Pont (D) & TN A42 & Arch. pers. \\
\hline 22 & Xanten (D) & TNC & Boelicke, 1995 et arch. pers. \\
\hline 23 & Karden (D) & TNA & Nickel, 1999 \\
\hline 24 & Kärlich (D) & TN A25 & Arch. pers. \\
\hline 25 & Urmitz (D) & TN A25 & Arch. pers. \\
\hline 26 & Xanten (D) & TN A43 & Arch. pers. \\
\hline 27 & Schanckweiler (D) & TN A41 & Ludwig, 1988 \\
\hline 28 & Karden (D) & TNA & Nickel, 1999 \\
\hline 29 & Karden (D) & TNA & Nickel, 1999 \\
\hline 30 & Nimègue (NL) & TN A44 & Holwerda, 1941 \\
\hline 31 & Karden (D) & TNA & Nickel, 1999 \\
\hline 32 & Karden (D) & TNA & Nickel, 1999 \\
\hline 33 & Karden (D) & TNA & Nickel, 1999 \\
\hline 34 & Xanten (D) & TN A43 (?) & Arch. pers. \\
\hline 35 & Xanten (D) & TNC & Ach. pers. \\
\hline 36 & Birten (D) & TN A25 & Ach. pers. \\
\hline 37 & Kärlich (D) & TN A8 & Arch. pers. \\
\hline
\end{tabular}

comportent également ( $\left.\mathrm{n}^{\mathrm{os}} 52,53,59\right)$. L'élément qui pourrait être commun à la plupart d'entre elles serait leur appartenance à des terra nigra et, mais rien n'est moins sûr, à des types plus récents (I'N A41-42).

\section{LA RHÉNANIE}

Si nous nous penchons sur la partie orientale de la Belgique ancienne, en particulier sur les cours inférieur de la Moselle, moyen et inférieur du Rhin, nous relevons une quinzaine d'ateliers ayant produit de la céramique belge. Peu d'entre eux fournissent du matériel estampillé : à titre d'exemple, les groupes d'ateliers de Cologne n'en connaissent pas ${ }^{245}$. En revanche, au vu des aires de répartition de certaines marques, il est certain que du matériel peut être attribué à cette région, sans que l'on soit en mesure de préciser les lieux de production (Deru, 1996b). C'est pourquoi nous privilégierons les produits de quatre ateliers : Metz, Trèves, Karden et Xanten.

\section{LES ATELIERS ET LES PRODUITS}

L'atelier de Saint-Pierre-aux-Nonnains à Metz fut découvert durant la Seconde Guerre mondiale et publié dans la foulée (Reusch, 1943). Un seul four fut mis au jour. À Trèves, il semble

245. Le mobilier des fouilles anciennes a en grande partie disparu durant la Seconde Guerre mondiale, mais l'ensemble des vestiges vient de faire l'objet d'une étude globale (C. Höpken, comm. pers.). que les quartiers réservés au travail de la terre soient importants dès le $\mathrm{I}^{\mathrm{er}} \mathrm{s}$.; plus tard, la capitale trévire deviendra un pôle majeur de cet artisanat avec les productions de terre sigillée, de céramique engobéc et métallescente. Les publications font néanmoins défaut pour les productions du $\mathrm{I}^{\mathrm{er}} \mathrm{s}$. et nous restons tributaires de la publication des estampilles de 1938 par H. Koethe.

En aval de Trèves, Karden est une agglomération secondaire sur la rive gauche du Rhin (Wegner, 1990b). Les structures artisanales ne sont pas publiées, mais une étude récente traitant de la vaisselle d'un sanctuaire local permet de les connaître indirectement (Nickel, 1999). Bien plus au nord, sur le Rhin inférieur, l'agglomération précoloniale de Xanten n'a pas livré de structures de production, mais plusieurs lots de rebuts de cuisson. Pour ce dernier site, en plus d'études récentes, nous bénéficions d'une observation directe du mobilier (Liesen, 1994 ; Boelicke, 1995).

Nous pouvons associer les productions de Metz et Trèves d'une part et celles de Karden et Xanten d'autre part. En effet, dans les deux ateliers mosellans les estampilles sont principalement imprimées sur des assiettes en terra rubra et en terra nigra issues du répertoire de la terre sigillée italique : TR/TN A5, A7, A8, A31 et A37, ainsi que sur le type plus récent TN A41. Malgré les lacunes de la documentation, ces productions peuvent être datées de la première moitié du ${ }^{\mathrm{rr}}$ s. Les productions de Karden et de Xanten correspondent à un répertoire plus récent où les formes TN A24/25 et A42/44 dominent.

\section{LES ESTAMPILLES}

Comme en Champagne, l'estampillage concerne surtout des formes ouvertes ; des pots estampillés existent en Rhénanie, mais ceux que l'on pourrait attribuer à des productions régionales sont très rares (fig. 75).

Dans les deux centres urbains de la Moselle, nous avons affaire, selon les données disponibles, à des estampilles rectangulaires et littérales. Sur les six estampilles trévires différentes $\left(n^{\circ} 1-6\right)$ et l'unique messine $\left(n^{\circ} 7\right)$, nous en comptons cinq clairement lisibles, dont une seule double avec cadre, et deux dont la graphie est plus médiocre.

À Karden et à Xanten, en revanche, les formes sont plus variées, rectangulaires, circulaires, in planta pedis et sont nonlittérales ou de graphies sommaires. Tout d'abord, nous avons affaire à des estampilles où des caractères alphabétiques sont reconnus, $\mathrm{A}, \mathrm{E}, \mathrm{I}, \mathrm{N}, \mathrm{O}, \mathrm{S}$ et $\mathrm{V}$, sans que l'on sache, à cause de leur graphie sommaire, $s$ 'ils présentent une valeur alphabétique $\left(n^{\text {os }} 8-11\right)$. Pour certaines, on reconnaît une logique d'abréviation ( $\mathrm{n}^{\circ \mathrm{s}} 8-10$ ). À Karden, nous pouvons classer des estampilles dans un premier groupe à partir de l'emploi de signes numériques $\mathrm{I}, \mathrm{V}$ et $\mathrm{X}$, sans que ces données aient une valeur quantitative ( $\left.n^{\text {os }} 12-16\right)$. Ailleurs en Rhénanie, nous trouvons d'autres estampilles associant ces mêmes signes ( $\left.n^{\text {os }} 17-21\right)$.

Des estampilles rectangulaires de Xanten montrent un simple quadrillage ( $n^{\circ} 22$ ), à Karden un motif de palme ( $n^{\circ} 23$ ) que l'on rencontre également ailleurs dans la région $\left(\mathrm{n}^{\text {os }}\right.$ 24-26). Les estampilles circulaires de Karden, les plus nombreuses dans le lot (60 ex. sur 123), n'illustrent que des étoiles formées de 4,5 , jusqu'à 12 rayons (n²8-29). 


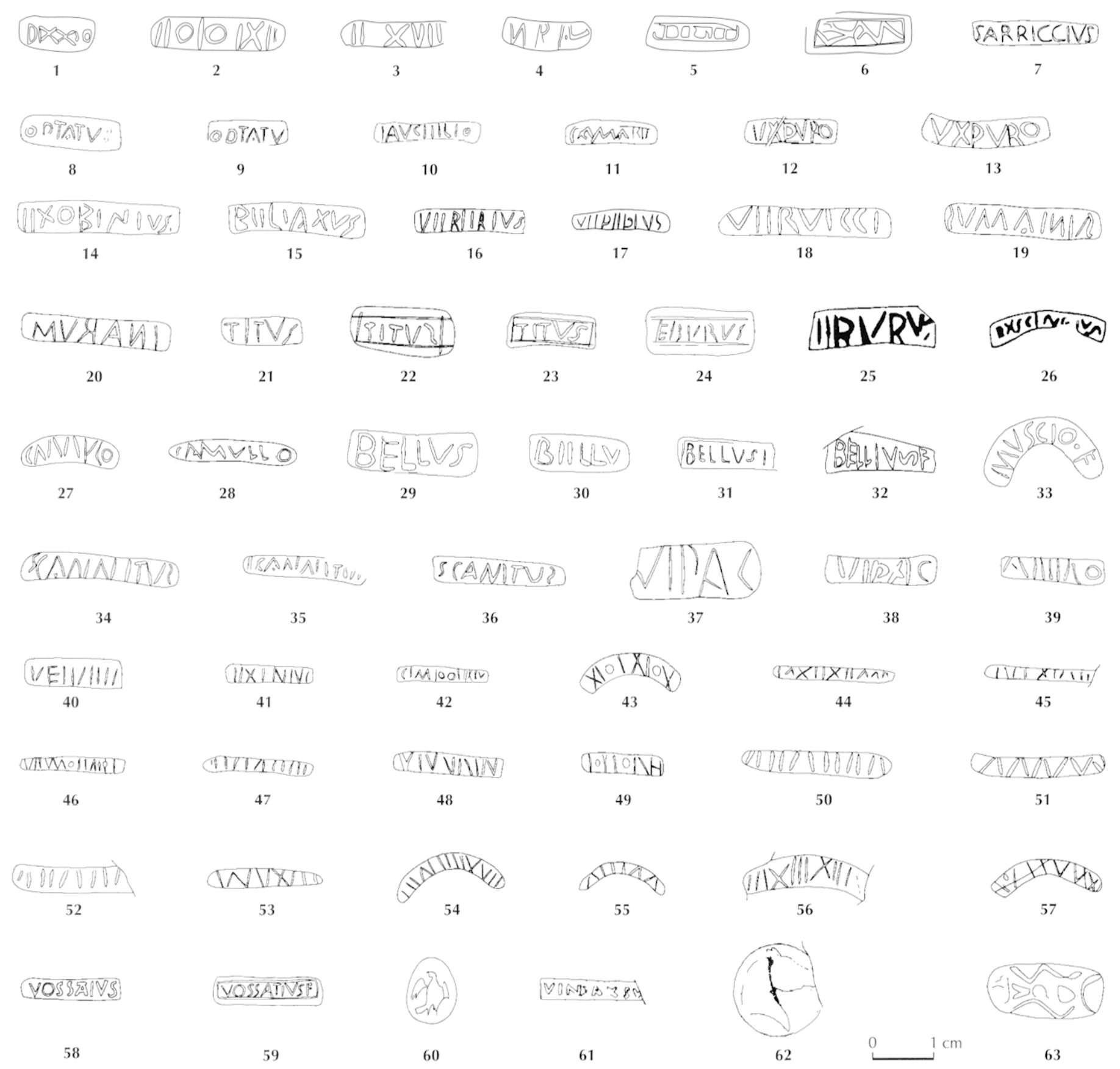

Fig. 76 - Estampilles sur terra rubra (TR), terra nigra (TN) et céramique dorée (I)R) fabriquées dans le Nord (cf. tableau, p. 141).

Les estampilles in planta pedis forment une spécificité de la Rhénanie ${ }^{2: 46}$. Un seul type est épigraphique (n³0). Bien qu’on n'en connaisse pas la provenance précise, il s'agit, d'après son aire de répartition (15 occurrences), d'un produit rhénan. À Karden, les estampilles plantaires montrent des traits (n $\left.{ }^{\circ 3} 31-33\right)$, alors que celles de Xanten présentent des points ( $\left.n^{*} 34-35\right)$; ailleurs on trouve aussi des signes numériques ( $n^{\text {os }} 36-37$ ).

246. Sur trente-neuf commues, une seule fut trouvée en dehors de la Rhénanie, à Bavay; cette dernière est également littérale et inconnue ailleurs.

\section{LE NORD}

Dans la partic nord occidentale de la Gaule belgique, de nombreux ateliers de céramique belge furent mis au jour. Rares sont ceux ayant livré du matériel estampillé. C'est un fait intéressant en tant que tel, cependant dans le cadre de cet article nous nous pencherons sur une région réduite, la partie méridionale de la civitas nerviorum. Après avoir évoqué du matériel caractérisé par une pâte particulière mais mal localisée faute d'avoir retrouvé des centres de production, nous nous pencherons sur les ateliers de Bavay et des Rues-des-Vignes. 


\begin{tabular}{|c|c|c|c|}
\hline \multicolumn{4}{|c|}{ FIGURE 76 (suite) } \\
\hline $\mathbf{N}^{\circ}$ & Site (Pays) & Forme & Bibliographie \\
\hline 1 & Bavay (F) & TN A42 & Loridant, Deru, à paraître \\
\hline 2 & Bavay (F) & TN C18 & Loridant, Deru, à paraitre \\
\hline 3 & Bavay (F) & TR1a a31 & Loridant, Deru, à paraître \\
\hline 4 & Bavay (F) & TN A42 & Loridant, Deru, à paraître \\
\hline 5 & Liberchies (B) & TN A42 & Brulet, Demanet dir., 1997 \\
\hline 6 & Bavay (F) & TN A42 & Loridant, Deru, à paraittre \\
\hline 7 & Bavay (F) & TN P & Arch. pers. \\
\hline 8 & Bavay (F) & TNP & Arch. pers. \\
\hline 9 & Bavay (F) & TNP & Arch. pers. \\
\hline 10 & Rues-des-Vignes (F) & TNP & Arch. pers. \\
\hline 11 & Rues-des-Vignes (F) & TN P & Arch. pers. \\
\hline 12 & Rues-des-Vignes (F) & TNP & Arch. pers. \\
\hline 13 & Bavay (F) & TNP & Arch. pers. \\
\hline 14 & Bavay (F) & TNP & Arch. pers. \\
\hline 15 & Bavay (F) & TNP & Arch. pers. \\
\hline 16 & Rues-des-Vignes (F) & TNP & Arch. pers. \\
\hline 17 & Braives (B) & DR DOR 2,22 & Brulet dir., 1985 \\
\hline 18 & Bavay $(F)$ & TNP & Arch. pers. \\
\hline 19 & Bavay (F) & TNP & Arch. pers. \\
\hline 20 & Bavay (F) & TN P & Arch. pers. \\
\hline 21 & Bavay (F) & TNP & Arch. pers. \\
\hline 22 & Nimègue (NL) & TN P & Arch. pers. \\
\hline 23 & Bavay (F) & TNP & Arch. pers. \\
\hline 24 & Bavay (F) & TNP & Arch. pers. \\
\hline 25 & Nimègue (NI) & DR DOR 2, 18 & Stuart, 1977 \\
\hline 26 & Nimègue (NL) & DR DOR 2,18 & Stuart, 1977 \\
\hline 27 & Bavay (F) & TN P & Arch. pers. \\
\hline 28 & Bavay (F) & TNP & Arch. pers. \\
\hline 29 & Bavay (F) & TN P & Arch. pers. \\
\hline 30 & Bavay $(\mathrm{F})$ & TNP & Arch. pers. \\
\hline 31 & Bavay (F) & TN P & Arch. pers. \\
\hline 32 & Kerben (D) & TN P54 & Arch. pers. \\
\hline 33 & Bavay (F) & TN P & Arch. pers. \\
\hline 34 & Bavay (F) & TN P & Arch. pers. \\
\hline 35 & Bavay (F) & TNP & Arch. pers. \\
\hline 36 & Bavay (F) & TNP & Arch. pers. \\
\hline 37 & Bavay (F) & TNP & Arch. pers. \\
\hline 38 & Bavay (F) & TNP & Arch. pers. \\
\hline 39 & Bavay (F) & TN P & Arch. pers. \\
\hline 40 & Bavay (F) & TNP & Arch. pers. \\
\hline 41 & Bavay (F) & TN P & Arch. pers. \\
\hline 42 & Bavay (F) & TNP & Arch. pers. \\
\hline 43 & Bavay (F) & TN P & Arch. pers. \\
\hline 44 & Bavay (F) & TNP & Arch. pers. \\
\hline 45 & Bavay $(F)$ & TN P & Arch. pers. \\
\hline 46 & Bavay (F) & TNP & Arch. pers. \\
\hline 47 & Bavay (F) & TN P & Arch. pers. \\
\hline 48 & Rues-des-Vignes (F) & Cachet & Arch. pers. \\
\hline 49 & Rues-des-Vignes (F) & Cachet & Arch. pers. \\
\hline 50 & Bavay (F) & TN P & Arch. pers. \\
\hline 51 & Bavay (F) & TNP & Arch. pers. \\
\hline 52 & Bavay $(\mathrm{F})$ & TNP & Arch. pers. \\
\hline 53 & Bavay (F) & TN P & Arch. pers. \\
\hline 54 & Rues-des-Vignes (F) & TNP & Arch. pers. \\
\hline 55 & Bavay (F) & TN P & Arch. pers. \\
\hline 56 & Bavay (F) & TNP & Arch. pers. \\
\hline 57 & Bavay (F) & TN P & Arch. pers. \\
\hline 58 & Bavay $(\mathrm{F})$ & TN P54 & Loridant, Deru, à paraître \\
\hline 59 & Reims (F) & TN P54 & Deru, Rollet, 2000 \\
\hline 60 & $\operatorname{Reims}(F)$ & TN P54 & Deru, Rollet, 2000 \\
\hline 61 & Saint-Quentin (F) & TNP & Clotuche, comm. pers. \\
\hline 62 & Saint-Quentin (F) & TN P & Clotuche, comm. pers. \\
\hline 63 & Bavay (F) & TN P & Arch. pers. \\
\hline
\end{tabular}

\section{LES ATELIERS ET LES PRODUITS}

La terra nigra fabriquée dans une argile calcaire, que l'on appelle du " groupe de pâtes savonneuses " (TN-SAVO), rassemble un répertoire diversifié compor- tant assiettes, pots et bouteilles datant de la seconde moitié du ${ }^{\mathrm{er}} \mathrm{s}$. (Deru, Vachard, 2002). D'après les analyses minéralogiques, les ateliers devraient se trouver au nord de Bavay.

Les ateliers de Bavay, quant à eux, firent l'objet d'observations anciennes et superficielles lors de l'exploitation des sablières situées au sud de la ville, sur la route de Vermand. Le mobilier estampillé conservé au musée départemental fait l'objet d'un réexamen complet (environ $450 \mathrm{ex}$.). Ce qu'on attribue à Bavay n'est donc pas entièrement fiable, vu les circonstances des fouilles et à cause d'un groupe de pâtes relativement hétérogène. Néanmoins, on le traitera comme un ensemble, faute de nouveaux arguments. L'atelier rural des Rues-des-Vignes est localisé plus au sud, sur la voie menant de Cambrai à Vermand. Ce site fait l'objet d'une fouille programmée depuis 1999 (Deru, à paraître). En activité pendant deux siècles à partir du dernier quart du $\mathrm{I}^{\mathrm{cr}} \mathrm{s}$., la terra nigra n'y est fabriquée qu'au cours de la première phase de production, jusqu'à 120 environ. Associée à ces productions, nous avons également identifié de la céramique dorée (DR), c'est-à-dire des récipients revêtus d'un engobe micacé, estampillée. Les plats à vernis rouge pompéien, qui forment la plus grande part des productions de l'atelier, ne portent pas d'estampilles, bien qu'un exemplaire marqué pouvant être attribué à cet atelier fût retrouvé sur un site de consommation.

Les estampilles appartenant aux terra nigra en "pâte savonneuse " sont toutes apposécs sur des formes ouvertes, TN A31 (?), A42 ou C18, datées du dernier tiers du $\mathrm{I}^{\mathrm{cr}} \mathrm{s}$. apr. J.-C. Les céramiques estampillées que l'on attribue à Bavay et celles fabriquées aux Rues-des-Vignes rassemblent surtout des formes fermées P45, P46, P48-49, P51, P54, P55 et P56. Ces récipients datent de la seconde moitié, même du dernier tiers, du $\mathrm{I}^{\mathrm{er}}$ s. apr. J.-C.

\section{LES ESTAMPILLES}

Sur la quinzaine d'estampilles identifiées sur les céramiques à "pâte savonneuse ", la majorité, si ce n'est la totalité, sont anćpigraphes (fig. 76). Certaines sont clairement gravées, mais présentent des signes simples I, X, O, V ( $\left.{ }^{\prime \prime \prime} 1-3\right)$, d'autres sont

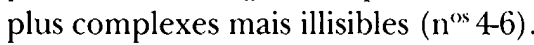

Les estampilles du groupe de Bavay et celles des Rues-desVignes sont apposées à la périphérie des fonds des gobelets. L'espace est réduit, ce qui explique que la plupart sont de petites tailles et qu'il n'en existe pas de circulaires, ni à double ligne. En revanche, un cinquième d'entre elles sont courbes et s'adaptent à la forme du pied ( $\left.\mathrm{n}^{\mathrm{os}} 26,33,43,54-57\right)$.

Les trois quarts des estampilles du groupe de Bavay sont littérales; le nombre d'estampilles retrouvées aux Rues-desVignes est encore trop insuffisant pour donner une proportion. Certaines présentent une graphie claire, mais comportent très rarement un cadre. Aucun nom n'est associé au verbe avotis; $F\left(\right.$ ecit) est également très sporadique ( $\left.n^{\circ 3} 32,33,59\right)$. Les simplifications de lettres sont régulières, mais touchent principalement la lettre E, remplacée par II ( ${ }^{\circ s} 10,14-18,30$, etc.). Si, comme en Champagne, les estampilles rétrogrades restent rares, certaines lettres, $\mathrm{R}, \mathrm{N}$ et $\mathrm{S}$, sont écrites à l'envers $\left(\mathrm{n}^{\circ} 19\right.$, $20,22,34,36$ ). Outre ces simplifications, certains noms présentent des variantes graphiques, ajout (ou retrait) d'un I à Bellus, d'un N à Scanitus, d'un Là Camulo (n" 27-28, 29-32, 34-36). Lne seule ligature est connue, elle est due à un potier fabriquant des gobelets aux parois à coquille d'œuf : Vossatius (n'58).

On peut classer les estampilles non littérales en plusieurs ensembles. Tout d'abord, des estampilles qui associent des caractères alphabétiques à des traits simples ne peuvent être 
considérées comme des simplifications de lettre ( $\left.\mathrm{n}^{\text {os }} 39-40\right)$. Ensuite, nous pouvons définir un groupe qui rassemble des estampilles longues et étroites où l'on retrouve caractères et traits ( $n^{\text {os }} 41-49$ et 54-55). La petitesse de ces estampilles nuit à leur lecture et le nombre important de caractères me font penser qu'elles ne sont pas littérales. Aux Rues-des-Vignes fut retrouvé un cachet en os avec deux estampilles de ce type (n $\left.{ }^{\circ} 48-49\right)$. Il s'agit d'un losange tronqué, épais de $7 \mathrm{~mm}$ et de $3 \mathrm{~mm}$, entaillé sur ses deux extrémités. Le trou qui le perce au centre témoigne d'une suspension à l'aide d'une ficelle. Cet objet personnel porte deux marques différentes montrant ainsi que des estampilles non littérales peuvent être abrégées, pour sans doute être adaptées à la surface disponible. Pour finir, un groupe d'estampilles ne présente que des traits sans qu'aucun caractère puisse être identifié ( $\left.\mathrm{n}^{\circ \varsigma} 50-53,56-57\right)$.

Dans la région septentrionale, on trouve une autre spécificité : celle de l'association sur le fond des pots d'une estampille littérale et d'une estampille figurée, peut-être l'impression d'une intaille. Ainsi, on connaît la représentation d'un aigle associé à l'estampille de Vossatius, plusieurs types de protomé de cheval associés au nom d'Atiolus, Bellius, Uxpuro, Vindac et Vindaxsus ( $\mathrm{n}^{\circ 5}$ 59-60, 61-62). Certains cachets figurés sont isolés, mais cela peut être dû à la conservation du récipient ( $\left.n^{\circ} 63\right)$.

Nous avons signalé des céramiques dorées estampillées. Elles comportent des estampilles identiques ou identifiant des mêmes potiers que ceux de la terra nigra $\left(\mathrm{n}^{\circ \mathrm{s}} 14,25,26\right)$. On a suggéré que les formes récentes de pots en terra nigra et celles de la céramique dorée étaient issues de la vaisselle en métal (Deru, 1994 et 1996a, p. 208). La mode de l'estampillage de ces récipients dépendrait des usages de l'orfèvrerie qui, aussi bien pour les formes que pour les modes d'estampillage, ne sont connus que superficiellement.

\section{$*$}

Les potiers qui marquent leurs produits d'estampilles, qu'elles soient épigraphiques ou non, font référence à la civilisation de l'écrit. On ne peut pourtant dire qu'ils y soient intégrés, encore moins qu'ils la maîtrisent, quant bien même les comptes de potiers de La Graufesenque (Marichal, 1988; Bémont, supra, p. 103-131) ou les contrats de travail conservés dans le Fayoum (Cockle, 1981). Ces potiers, de même que les autres artisans et les paysans, bien qu'appartenant à une civilisation du droit et de la comptabilité, restent cantonnés à la périphérie de la culture de l'écrit. L'ethnographie et la sociologie, ainsi que de manière indirecte les épitaphes antiques, pointent une réalité que les sources nous occultent où les classes sociales pauvres et fortement majoritaires n'ont pas accès à l'écrit, mais seulement et quelquefois au déchiffrement (Duncan-Jones, 1977 et 1990 ; Kunow, 1983 ; Hanson, 1991).

La qualité des estampilles, sans tenir compte de leur alphabétisation, peut néanmoins être un indice de la proximité avec cette culture. Clarté des signes, empattement et ligature des lettres, cartouches, à la différence des simplifications et des rétrogadations de caractères, peuvent former des critères, si l'épigraphie monumentale est la norme, pour la qualification des estampilles. La Champagne regroupe dès lors le plus grand nombre de critères de qualité et d'estampilles rédigées selon ceux-ci ; dans le Nord, si ces critères sont quelquefois présents, ils demeurent sporadiques.

Le caractère alphabétique doit maintenant être appréhendé selon notre point de vue géographique et chronologique caractérisant a priori des groupes sociaux d'artisans. La capitale de la Belgique ancienne, Reims, et son arrière-pays regroupent les ateliers où l'on marque habituellement la vaisselle, presque exclusivement des assiettes et des coupes. Seules $15 \%$ des estampilles sont anépigraphes. D'après l'onomastique, les potiers sont Gaulois, mais à partir de leurs produits on constate qu'ils maîtrisent des techniques (tournage, mouluration, engobage, cuisson) et un répertoire (imitation de terre sigillée, module, etc.) fortement romanisés. J'ai dès lors pensé que les ateliers qui fleurissent en Champagne à la période augustéenne correspondent à la venue volontaire ou non d'artisans de Cisalpine ou de Narbonnaise. Des entrepreneurs les installeraient chez les Rèmes, comme investissement complémentaire à l'exploitation d'un terroir rapidement pacifié et nouvellement intégré à l'Empire (Deru, 1996a, p. 237). Ces potiers témoignent donc d'un artisanat proche de la culture de l'écrit méditerranéenne. Une hypothèse identique peut être proposée pour les potiers $d$ ' «imitations de terre sigillée helvétique " ${ }^{247}$ et d'imitations de terre sigillée languedocienne (Passelac, 1992 et 2001 ; Lüginbuhl, 2001, p. 294 et passim).

En Rhénanie, les estampilles littérales sont rares et mettent en lumière de nouveaux facteurs : il s'agit des ateliers de Metz et de Trèves, deux ateliers précoces et urbains. On pourrait les comprendre grâce au premier modèle, celui d'un transfert de personnel dans ces pôles. Concernant la masse d'estampilles non littérales, il faut d'abord souligner que la proximité des marchés militaires, contrairement à ce que l'on aurait pu croire, n'a pas joué en faveur d'une plus grande intégration des potiers dans la culture de l'écrit. En effet, les effectifs de la céramique belge à l'intérieur des camps sont faibles et montrent dès lors une claire distinction entre les productions de cette vaisselle et ces marchés. On pourrait alors déduire du matériel portant des estampilles non littérales qu'existe entre les premiers potiers de Metz et de Trèves et leurs successeurs une rupture sociale: la deuxième génération appartiendrait plus à la culture indigène, analphabète. Nous ne le pensons pas car, d'une part, l'estampillage non littéral témoigne de toute manière de la culture de l'écrit et, d'autre part, la typologie (A24/25, A43 en particulier) et les formes d'estampilles (in planta pedis ou avec palme) montrent que ces potiers étaient au fait des courants affectant la terre sigillée italique postérieure à 10 apr. J.-C: Nous pourrions suggérer simplement qu'une distance géographique, chronologique et donc sociale et culturelle, avec les foyers de production, qu'ils soient champenois ou méridionaux, a conduit à la perte de l'écrit, du contenu alors que la forme est préservée. Ce modèle se retrouve pour les productions de terre sigillée italique. Si les estampilles non littérales sont absentes dans les centres de production principaux, à Arez,o, Pisc ou I yon, elles se retrou-

247. T. Lüginbuhl privilégie néanmoins l'hypothèse selon laquelle la plupart des potiers de sigillée helvétique étaient indigènes. 
vent dans des ateliers périphériques comme à Pouzzoles ou Scoppieto (Ombrie) ou dans la vallée du Pô (Oxé et al., 2000, p. 25-35 et p. 513-523; Nicoletta, 2003). Dans la seconde moitié du ${ }^{\mathrm{er}}$ s., l'est de la Gaule connaîtra une deuxième rupture chez les producteurs de vaisselle par l'implantation de fabricants de terre sigillée, de leur nouvelle technique, répertoire et estampilles épigraphiques (Hoerner, 2000).

L'estampillage dans le nord, quant à lui, se partage en deux phénomènes distincts. L'estampillage sur la céramique à " pâte savonneuse " où les marques sont non littérales témoigne vraisemblablement du même phénomène qu'en Rhénanie ou dans les régions périphériques de l'Italie : une distance par rapport au cœur, par laquelle l'écrit est perdu, bien avant la technique ou le répertoire. Le deuxième phénomène est également tardif, du dernier tiers du I ${ }^{\mathrm{er}} \mathrm{s}$., et n'est plus lié à la terre sigillée, mais sans doute à des récipients en métal. Dans les ateliers de Bavay et des Rues-des-Vignes, un quart des estampilles sont non littérales ; elles pourraient être plus fréquentes dans le second atelier, rural et plus récent. Par rapport à l'environnement culturel où l'écrit est moins développé que chez les Rèmes, les Trévires et les populations de l'est (Wightman, 1985 p. 162177), ce phénomène est particulier et pourrait faire surgir un nouveau critère d'analyse, que l'on aurait déjà pu proposer pour le cas champenois. Il s'agit de la qualité des produits. Les pots biconiques (TN P54) peuvent servir la démonstration. Ils sont fabriqués à travers toute la Gaule septentrionale, mais ceux du nord présentent la meilleure qualité, des surfaces d'un noir profond et des parois d'une grande finesse, c'est-à-dire des récipients très légers. Ces gobelets bénéficient d'un estampillage, généralement alphabétique. Il pourrait donc y avoir une convergence entre technicité et culture de l'écrit.

En Gaule septentrionale, l'illettrisme devait être la norme, l'artisanat ne déroge pas à la règle. L'estampillage alphabétique d'une partie du répertoire de certaines catégories de céramique correspond donc à des épiphénomènes, mais il témoigne, comme d'ailleurs les estampilles anépigraphes, d'une civilisation de l'écrit. Ces épiphénomènes peuvent se comprendre à travers plusieurs interprétations. Tout d'abord, les artisans, peu importe leur statut, qui possèdent la plus haute technicité forment des groupes sociaux plus proches de la culture de l'écrit. Nous l'avons observé en Champagne et dans les ateliers urbains de l'est, ensuite pour les ateliers de Bavay et des Rues-des-Vignes. Pour les premiers ateliers, les productions de céramique belge se réfèrent aux productions de terre sigillée et donc directement à la culture méditerranéenne. Un simple transfert de groupes sociaux du sud vers le nord pourrait expliquer cet épiphénomène. Dans les ateliers du nord, la référence artisanale est différente, ce n'est plus par imitation de la terre sigillée que l'on estampille, mais vraisemblablement par rapport à la vaisselle en métal. Ces ateliers deviennent des foyers d'excellence vis-à-vis desquels d'autres travailleront. La deuxième génération de potiers ayant produit de la céramique belge estampillée dans l'est et ceux ayant produit la terra nigra à " pâte savonneuse " n'ont que peu perdu de la technicité précédente, mais néanmoins montrent unc simplification de celle-ci et des formes; en revanche, ils se sont éloignés de l'écrit tout en y faisant référence au moyen d'estampilles anépigraphes. Un environnement rural, une exigence de qualité moindre et une plus faible ambition commerciale témoignent et expliquent à la fois cette plus grande distance par rapport à l'écrit.

En conclusion, le monde artisanal est à la périphérie de la culture de l'écrit. Pour des groupes sociaux déterminés par leur origine, la qualité de leur produit, la localisation de leur atelier, cette culture est plus prégnante et devient une référence explicite ; pour d'autres, cette référence n'est plus qu'implicite, tandis que pour la majorité des artisans elle n'existe pas.

\section{Nota bene}

Je remercie M. Feugère de m'avoir suggéré cette problématique, ainsi que toutes les personnes m'ayant fourni des documents épigraphiques. Je remercie en particulier A. Revelart pour les centaines d'estampilles redessinées. 